\title{
Thermal Dilepton Radiation at Intermediate Masses at the CERN-SpS
}

\author{
Ralf Rapp and Edward Shuryak \\ Department of Physics and Astronomy, State University of New York, Stony Brook, NY 11794-3800, U.S.A.
}

(July 2, 2021)

\begin{abstract}
We investigate the significance of thermal dilepton radiation in the intermediate-mass region in heavy-ion reactions at CERN-SpS energies. Within a thermal fireball model for the space-time evolution, the radiation from hot matter is found to dominate over hard 'background' processes (Drell-Yan and open charm) up to invariant masses of about $2 \mathrm{GeV}$, with a rather moderate fraction emerging from early stages with temperatures $T \simeq 175-200 \mathrm{MeV}$ associated with deconfined matter. Further including a schematic acceptance for the NA50 experiment we find good agreement with the observed enhancement in the region $1.5 \mathrm{GeV}<M_{\mu \mu}<3 \mathrm{GeV}$. In particular, there is no need to invoke any anomalous open charm enhancement.
\end{abstract}

\section{INTRODUCTION}

The identification of experimental signals from a possibly formed Quark-Gluon Plasma (QGP) constitutes one of the prime goals in the (ultra-) relativistic heavy-ion program. Among the earliest suggestions [i] for QGP signatures has been the appearance of an enhanced radiation of dileptons (and/or photons) in the intermediate-mass region (IMR), $1.5 \mathrm{GeV}<M_{l l}<3 \mathrm{GeV}$, i.e., well above the light vector mesons $\rho, \omega$ and $\phi$ but below the $J / \Psi$ resonances. The importance of this region resides on the facts that, on the one hand, low-energy hadronic processes (such as $\pi \pi$ annihilation or Dalitz decays) are sufficiently suppressed, and, on the other hand, hard processes (in particular Drell-Yan annihilation), which prevail in the high-mass region $M \geq 4 \mathrm{GeV}$, increase rather slowly towards smaller $M$ and thus may be over-shined by thermal radiation.

At the CERN-SpS, intermediate-mass dilepton spectra have been measured in the dimuon mode by the NA38/NA50 [2,3] and HELIOS-3 [4] collaborations (the dielectron data from the CERES experiment [5] lack sufficient statistics in this region). In central collisions of heavy nuclei both experiments found factors of $2-3$ enhancement over the extrapolation of known sources from proton-induced collisions, given by primordial Drell-Yan annihilation [6] as well as semileptonic decays of associatedly produced charmed $(D, \bar{D})$ mesons.

Several suggestions to explain this excess have been made, e.g., an enhanced production of $c \bar{c}$ pairs. However, such an increase is not easily justified theoretically [7], and would also be difficult to reconcile with the current understanding of the observed $J / \Psi$ suppression. Another possibility has been pursued by Lin and Wang [8] who showed that $D$-meson rescattering in hot/dense matter (without invoking anomalous enhancement) might generate a transverse momentum broadening which can enrich the $\mu^{+} \mu^{-}$phase space covered by the NA50 experiment. The resulting increase is, however, much too small to explain the data [3]. Spieles et al. [9] have investigated the role of secondary Drell-Yan processes in hadronic rescatterings within the UrQMD transport model and found that at an invariant mass of, e.g., $M_{\mu \mu}=1.5 \mathrm{GeV}$ this contribution may constitute up to $\sim 30 \%$ of the primordial Drell-Yan yield. This is again far from being able to account for the experimental findings. Finally, Li and Gale analyzed the IMR of the HELIOS-3 data using a transport model incorporating dilepton production through secondary hadronic annihilation processes [10]. They found that the enhancement in S+W collisions can indeed be explained; unfortunately, the HELIOS-3 data quickly run out of statistics for $M_{\mu \mu} \gtrsim 2 \mathrm{GeV}$. Since the transport framework employed was entirely based on hadronic degrees of freedom, a possible Quark-Gluon Plasma formation was not addressed explicitly.

Another important issue is the consistency with dilepton measurements in the low-mass region (LMR). The CERES/NA45 [5] and HELIOS-3 [4] collaborations have reported a significant excess over final-state hadron decays which can indeed be explained by thermal radiation once medium effects in the vector meson spectral functions (especially for the $\rho$ ) are accounted for [11,12] (see Ref. [13] for a recent review). In this letter we would like to investigate whether thermal production in the $1.5-3 \mathrm{GeV}$ region, folded over the same fireball evolution as employed for the LMR in Ref. [12], can simultaneously describe the NA50 data. In addition, by allowing for a possible QGP formation, we study the significance of dileptons produced through quark-antiquark annihilation in the early phases of central $\mathrm{Pb}(158 \mathrm{AGeV})+\mathrm{Pb}$ collisions. 


\section{DILEPTON SOURCES AT INTERMEDIATE MASSES}

In the absence of any medium (or rescattering) effects, dilepton production in hadronic collisions arises from either initial hard processes or the decay of produced mesons. At invariant masses beyond $1.5 \mathrm{GeV}$, the contribution from light hadron decays (containing $u, d$ and $s$ quarks) sharply drops off either due their insufficient mass (as, e.g., for $\rho, \omega$ and $\phi$ mesons) or because of their increasing total widths resulting in a small electromagnetic branching ratio (as, e.g., for the $\rho^{\prime}$ resonances). For masses between $1.5 \mathrm{GeV}<M<3 \mathrm{GeV}$ the most important decay contribution therefore arises from the associated decay of charmed mesons $D, \bar{D}$ originating from primordial production of a $c \bar{c}$ pair (the thermal abundance of $D$-mesons is negligible at SpS energies). The other significant 'background' source in the IMR is the well-known Drell-Yan (DY) process [6]. The latter entirely saturates the yield at high masses beyond $\sim 5 \mathrm{GeV}$ and can thus be exploited for normalization purposes as has been verified by the NA50 collaboration 14. To be able to compare our calculations to the NA50 data we will adopt the same strategy here (in addition, the shape of the DY-yield after applying acceptance cuts can be checked versus experimental simulations using event generators). In a central collision of two equal nuclei with mass number $A$ the number of produced Drell-Yan pairs per invariant mass and rapidity interval can be obtained as

$$
\frac{d N_{D Y}^{A A}}{d M d y}(b=0)=\frac{3}{4 \pi R_{0}^{2}} A^{4 / 3} \frac{d \sigma_{D Y}^{N N}}{d M d y}
$$

in terms of the (standard) elementary Drell-Yan cross section in a nucleon-nucleon collision,

$$
\frac{d \sigma_{D Y}^{N N}}{d M d y}=K \frac{8 \pi \alpha}{9 s M} \sum_{q=u, d, s} e_{q}^{2}\left[q\left(x_{1}\right) \bar{q}\left(x_{2}\right)+\bar{q}\left(x_{1}\right) q\left(x_{2}\right)\right]
$$

Here, $q\left(x_{1,2}\right)\left(\bar{q}\left(x_{1,2}\right)\right)$ denote the (anti-) quark distribution functions within a pair of colliding nucleons from the two incoming nuclei (neglecting nuclear effects). Their arguments are related to the center-of-mass rapidity $y$ and invariant mass of the lepton pair as $x_{1,2}=x e^{ \pm y}$ with $x=M / \sqrt{s}$, where $s$ denotes the total cms energy of the nucleon-nucleon collision. For the parton distribution functions we employ the recent leading order parameterization from Ref. [15] (=GRV-94 LO), which necessitates a $K$-factor of 1.5 [9] (accounting for higher order effects) to comply with $p$ - $A$ Drell-Yan data. As has been further pointed out in Ref. [9], the explicit inclusion of isospin asymmetric sea-quark distributions in the GRV-94 LO set entails substantially smaller isospin corrections for nuclear collisions; e.g., for $\mathrm{Pb}+\mathrm{Pb}$ the correction is as small as $3 \%$, which will be neglected in the following. In Eq. (1), the root-mean squared radius parameter $R_{0} \simeq 1.05 \mathrm{fm}$ arises from a folding over a Gaussian thickness function [16]. For (slightly) non-central collisions as considered below we simply replace $A$ in Eq. (1) by the number of participants.

In nuclear collisions additional so-called 'thermal' dileptons are radiated through interactions during the expansion/cooling of the hadronic fireball. In thermal equilibrium, the dilepton production rate depends on the temperature and (baryon-) density of the matter and is governed by the electromagnetic current-current correlator $\Pi_{\mathrm{em}}$. Whereas at low masses medium modifications of the light vector mesons (especially the $\rho$ ) lead to a strong reshaping of the correlator [12], the situation simplifies in the intermediate-mass region: already in vacuum the cross section for the reverse process of $e^{+} e^{-} \rightarrow$ hadrons is well-described by lowest-order perturbative annihilation into $q \bar{q}$ pairs; at the correspondingly probed distance scales of $d \leq 1 /(1.5 \mathrm{GeV}) \simeq 0.13 \mathrm{fm}$, additional medium effects are expected to be no longer relevant. In fact, as has been shown in Ref. [10], a rate calculation involving a large set of hadronic reactions fitted to reproduce exclusive $e^{+} e^{-} \rightarrow$ hadrons cross sections yields an equivalent dilepton production rate (within the experimental uncertainties), i.e., the hadronic and partonic description of $\Pi_{\mathrm{em}}$ are 'dual' for $M \geq 1.5 \mathrm{GeV}$. We will thus employ the simple partonic rate

$$
\begin{aligned}
\frac{d^{8} N_{\mu \mu}}{d^{4} x d^{4} q} & =\frac{\alpha^{2}}{4 \pi^{4}} f^{B}\left(q_{0} ; T\right) \sum_{q=u, d, s}\left(e_{q}\right)^{2} \\
& =\frac{\alpha^{2}}{4 \pi^{4}} f^{B}\left(q_{0} ; T\right) \frac{2}{3}
\end{aligned}
$$

and note that in the IMR both temperature and density corrections, being of order $O(T / M)$ and $O\left(\mu_{q} / M\right)$, are irrelevant under the conditions probed at the $\mathrm{SpS}$. To obtain the total yield from thermal radiation, the rate (3) has to be integrated over the space-time history of a given collision system. Here we employ a simplified thermal fireball description including, however, the most important features of a full hydrodynamic simulation in a transparent way. As delineated in Ref. 12 we first construct a trajectory in the baryon-chemical potential/temperature plane based on baryon-number and entropy conservation. Using a resonance gas equation of state (EoS) including the 32 
(16) lowest lying mesonic (baryonic) states with $s / \varrho_{B}=26$ it is found to be consistent with the recently determined chemical freezeout point of $\left(\mu_{B}, T\right)_{\text {chem }}=(270 \pm 20,175 \pm 10) \mathrm{MeV}$ [17, which we also assume to be the critical point separating the hadronic and quark-gluon phase. In the hadronic evolution towards thermal freezeout, $\left(T, \mu_{N}\right)_{f_{o}}=$ $(115 \pm 10,430 \pm 30) \mathrm{MeV}$, one additionally requires pion (kaon) number conservation to maintain the final pion-tobaryon ratio of 5:1 at SpS energies, entailing the build-up of finite pion (kaon) chemical potentials reaching $\mu_{\pi}=$ $60-80 \mathrm{MeV}\left(\mu_{K, \bar{K}}=100-130 \mathrm{MeV}\right)$. For the initial stages we assume an ideal QGP EoS. A time scale is introduced by employing a cylindrical hydro-type volume expansion

$$
V_{F C}=2\left(z_{0}+v_{z} t+\frac{1}{2} a_{z} t^{2}\right) \pi\left(r_{0}+\frac{1}{2} a_{\perp} t^{2}\right)^{2}
$$

with a forward and a backward going firecylinder [12] to accomodate a realistic rapidity spread in the final particle distributions. $r_{0}$ corresponds to the initial transverse overlap of the two colliding nuclei (determined by the impact parameter $b$ ), and the parameters $v_{z}, a_{z}$ and $a_{\perp}$ are adjusted to the finally observed flow velocities. Given the total entropy of the reaction, the value of $z_{0}$ is equivalent to a formation time (or initial temperature). Once the system has cooled down to $T_{c}$, the fraction $f$ of matter in the hadronic phase can be inferred from the standard entropy balance

$$
f s_{H G}\left(T_{c}\right)+(1-f) s_{Q G P}\left(T_{c}\right)=S_{t o t} / V_{F C}(t) .
$$

The time-integrated dilepton yield from one collision event then takes the form

$$
\frac{d N_{\mu \mu}^{t h e r m}}{d M}=\frac{2}{3} \frac{\alpha^{2}}{\pi^{3}} M \int_{0}^{t_{f o}} d t V_{F C}(t) \int_{0}^{\infty} d q \frac{q^{2}}{q_{0}} f^{B}\left(q_{0} ; T(t), \mu_{\pi}(t)\right) \operatorname{Acc}(M, q, y),
$$

where the factor $\operatorname{Acc}(M, q, y)$ simulates the experimental acceptance that the produced muons undergo in the NA50 detector, see below. In the hadronic phase, the Bose factor $f^{B}\left(q_{0} ; T(t), \mu_{\pi}(t)\right)$ includes the enhancement due to finite $\mu_{\pi}$ 's. Since in the IMR the vector correlator is dominated by $\pi a_{1}$ annihilation [10], which is a 4-pion type process, the $\mu_{\pi}=0$ value of the Bose factor is augmented by a factor $\sim \exp \left(4 \mu_{\pi} / T\right)$.

Before we come to the full results let us compare the relative importance of Drell-Yan versus thermal pairs without any cuts. Fig. 1 shows the total dilepton yields from Drell-Yan annihilation and the time-integrated thermal signal from a $b=1 \mathrm{fm} \mathrm{Pb}(158 \mathrm{AGeV})+\mathrm{Pb}$ collision. The latter has been calculated starting from an initial temperature $T_{i}=192 \mathrm{MeV}$ (corresponding to $z_{0}=2.3 \mathrm{fm}$ in Eq. (4i)) cooling down to about $T_{f o}=113 \mathrm{MeV}$ within a total fireball lifetime of $\sim 14 \mathrm{fm} / \mathrm{c}$. As expected, the thermal curve (solid line) dominates towards low invariant masses but drops below the DY component (dashed-dotted line) at $M \geq 1.8 \mathrm{GeV}$ due its much softer slope.

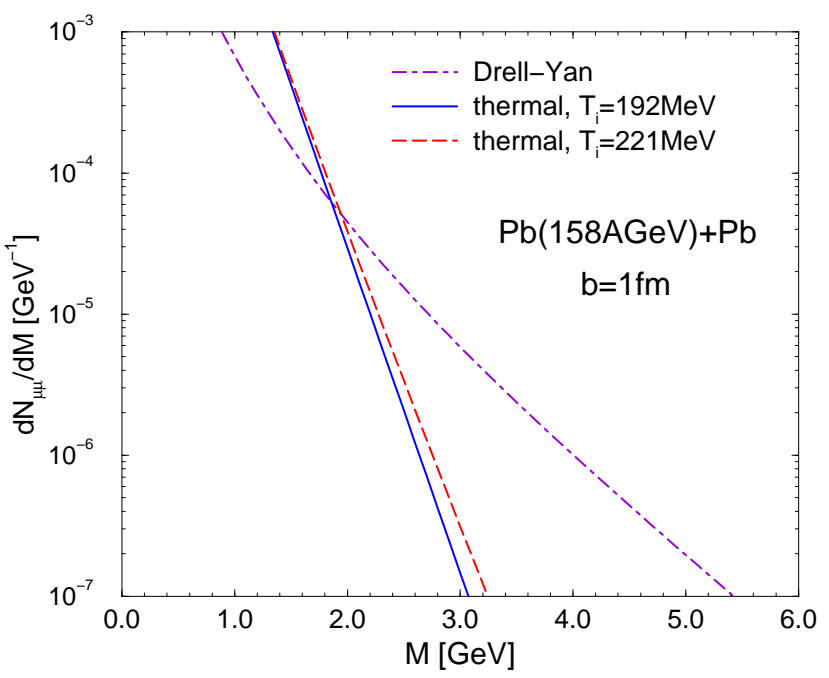

FIG. 1. Drell-Yan (dashed-dotted line) versus thermally produced $\mu^{+} \mu^{-}$pairs in central $\mathrm{Pb}(158 \mathrm{AGeV})+\mathrm{Pb}$, integrated over all rapidities and without any acceptance cuts. 
To test the sensitivity with respect to the initial conditions, we have carried out a calculation with $z_{0}=1.5 \mathrm{fm}$ which translates into an initial temperature $T_{i}=221 \mathrm{MeV}$. With the same fireball lifetime of $14 \mathrm{fm} / \mathrm{c}$ as before (leading to a practically identical freezeout temperature) the thermal spectrum looks rather similar to the $T_{i}=192 \mathrm{MeV}$ case up to $M \simeq 2 \mathrm{GeV}$, indicating that the decrease of the rate at smaller temperatures (corresponding to later stages) is essentially compensated by the larger volumes. Only at masses beyond $2 \mathrm{GeV}$ the higher initial temperature entails a visible increase of the thermal yield together with a slightly harder slope.

\section{IMR SPECTRA IN NA50}

In order to compare our calculations to the measurements of NA50 we have to account for the experimental acceptance cuts. In the IMR the geometry of the spectrometer imposes kinematic restrictions which are approximately given by [18]

$$
\begin{aligned}
0 & \leq y_{\mu \mu}^{c m s} \leq 1 \\
-0.5 & \leq \cos \Theta_{C S}^{+} \leq 0.5,
\end{aligned}
$$

where $y_{\mu \mu}^{c m s}$ is the pair rapidity in the $c m s$ of the colliding nuclei and

$$
\cos \Theta_{C S}^{+}=\frac{p_{z}^{+}-\beta_{z}^{\text {pair }} E^{+}}{\left[\left(1-\beta_{z}^{\text {pair }}{ }^{2}\right)\left(M^{2} / 4-m_{\mu}^{2}\right)\right]^{1 / 2}} .
$$

Here, $\Theta_{C S}^{+}$denotes the polar angle of the $\mu^{+}$with respect to the $z$-axis in the rest frame of the dimuon (the so-called Collins-Soper frame) with $p_{z}^{+}, E^{+}$and $\beta_{z}^{\text {pair }}=\left(p_{z}^{+}+p_{z}^{-}\right) /\left(E^{+}+E^{-}\right)$measured in the laboratory frame. Furthermore, the single-muon tracks in the lab-frame are selected according to

$$
0.037 \leq \Theta^{\mu} \leq 0.108 \text {. }
$$

More difficult to asses are absorption effects on the single-muon tracks; as a very rough characterization thereof we require the single-muon energies to satisfy [18]

$$
E_{\mu}> \begin{cases}E_{\text {cut }}+16000\left(\Theta_{\mu}-0.065\right)^{2} & , 0.037 \leq \Theta_{\mu} \leq 0.065 \\ E_{\text {cut }} & , 0.065 \leq \Theta_{\mu} \leq 0.090 \\ E_{\text {cut }}+13000\left(\Theta_{\mu}-0.090\right)^{2} & , 0.090 \leq \Theta_{\mu} \leq 0.108,\end{cases}
$$

where $E_{c u t}$ has been chosen as $8 \mathrm{GeV}$ in Ref. [8]. Following Refs. [19].3], we have also incorporated a phenomenological $q_{t}$-broadening of the Drell-Yan spectra by supplying Eq. (1) with a Gaussian factor $\exp \left(-q_{t}^{2} / 2 \sigma_{q_{t}}^{2}\right) / 2 \sigma_{q_{t}}^{2}$ to obtain $d N_{D Y}^{A A} / d M d y d q_{t}^{2}$ with $\sigma_{q_{t}}=0.8-0.9 \mathrm{GeV}$. Finally, we account for the finite experimental mass resolution by folding the calculated spectra with a Gaussian, the effective $\sigma_{M}$ being estimated from the apparent width of the $J / \Psi$ peak.

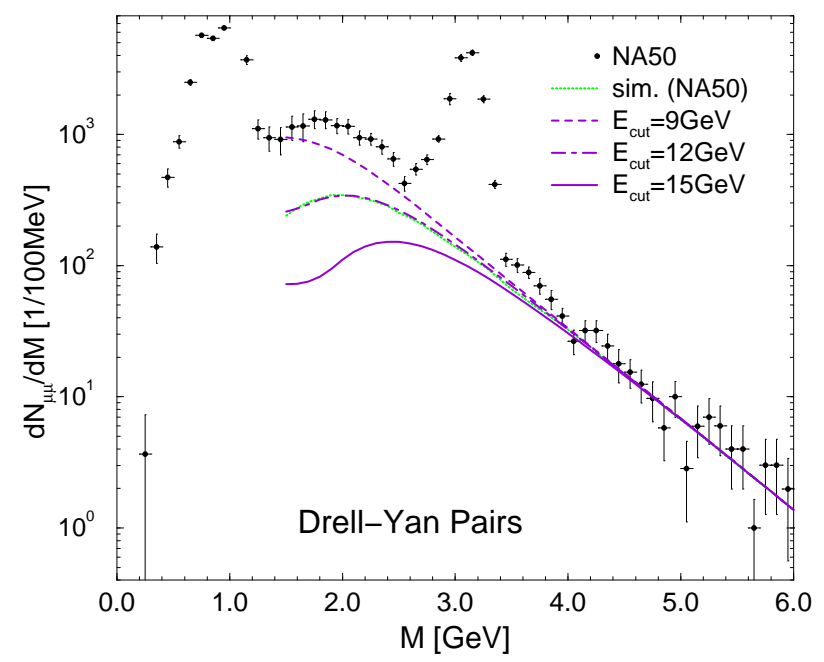

FIG. 2. Effects of the schematic cuts, Eqs. (7) and (10), on the invariant mass spectrum of Drell-Yan produced pairs in central $\mathrm{Pb}(158 \mathrm{AGeV})+\mathrm{Pb}$. In comparison to a detector simulation of the NA50 collaboration (dotted line; cf. also Figure 1 in Ref. 19] or the Drell-Yan fits shown in Ref. [3])) the curve calculated with $E_{c u t}=12 \mathrm{GeV}$ seems to give the best representation of the experimental acceptance. 
We have checked the schematic acceptance by comparing our results for the Drell-Yan production with and without the cuts to a simulation of the NA50 collaboration using the PYTHIA event generator [19]. The pertinent results are displayed in Fig. 2 using three different values for the single-muon energy threshold. The latter does not significantly affect the high-mass tail beyond $4 \mathrm{GeV}$ which has been used to fix the normalization in comparison to the NA50 data. From the behavior towards lower masses it seems that $E_{c u t} \simeq 12 \mathrm{GeV}$ gives the closest reproduction of the simulation results from the NA50 collaboration 19.3]. We will therefore use this value in the following also for the thermal radiation contribution, but note that this might not be an accurate representation of the actual experimental situation.

To enable a more complete comparison with the NA50 data, the associated decays of open charm mesons have to be included. We simply adopt this contribution as given in Ref. [3] (without any anomalous enhancement factor).

In Fig. 3 our final results are confronted with the NA50 data in central $\mathrm{Pb}+\mathrm{Pb}$ collisions at $158 \mathrm{AGeV}$ projectile energy. Given the uncertainties in the simulation of the experimental acceptance, the excess observed in the IMR $(1.5 \mathrm{GeV}<M<2.5 \mathrm{GeV})$ can be well accounted for by thermal radiation when combined with Drell-Yan and open charm pairs (resulting in the full line in the left panel). Also the shape and magnitude of the transverse momentum spectra (right panel of Fig. 3) are rather well described once thermal pairs are included.
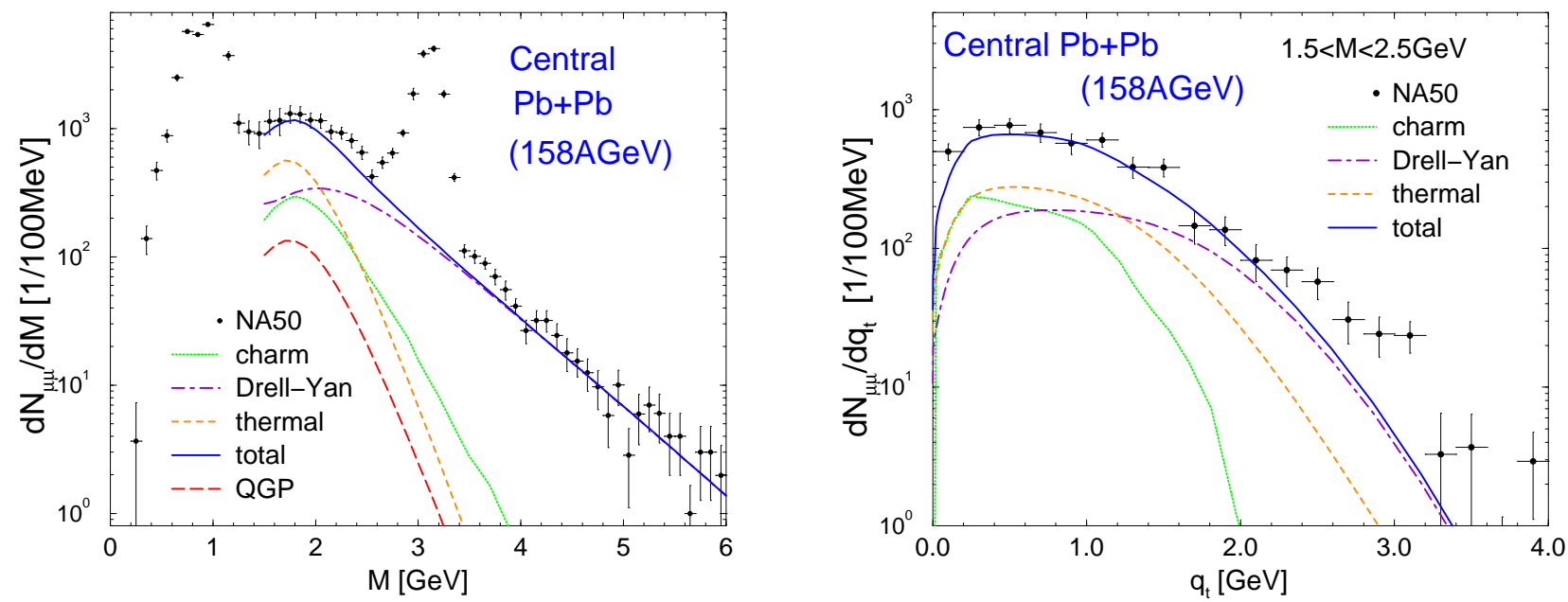

FIG. 3. IMR dilepton spectra in comparison to NA50 data from central $\mathrm{Pb}(158 \mathrm{AGeV})+\mathrm{Pb}$ collisions with $N_{\text {part }} \simeq 380$ (left panel: invariant mass spectra, right panel: transverse momentum spectra). The short-dashed and dashed-dotted curves are the calculated thermal and Drell-Yan yields, respectively, using the approximate acceptance as given in the text. The long-dashed curve in the left panel is the part of the thermal contribution originating from the plasma phase. The dotted curves represent the open charm contribution as obtained by the NA50 collaboration in a PYTHIA simulation without anomalous enhancement 3 . The full lines are the sum of thermal, open charm and Drell-Yan dileptons.

As mentioned in the introduction an important issue in the IMR is the quantitative role of radiation from the QGP. With our standard value for the initial temperature of $T_{i}=192 \mathrm{MeV}$, the $q \bar{q}$ contribution to the thermal yield constitutes about $25 \%$ (slightly more if a higher $T_{i}$ is assumed). This is significantly more than typical estimates for the low-mass region, but still moderate owing to the rather small space-time volume occupied by a QGP at the (full) SpS energies. Although perturbative $\alpha_{s}$-corrections might enhance the partonic production rate somewhat the major signal will still originate from the hadronic phase.

One may further ask to what extent the presence of finite pion chemical potentials influences the thermal yield. When using $\mu_{\pi}=0$ throughout the latter is reduced by about 30-40\% (we should stress, however, that such a calculation underestimates the measured pion multiplicity in central $\mathrm{Pb}(158 \mathrm{AGeV})+\mathrm{Pb}$ by $\sim 40-50 \%)$. Thus, similar to what has been found in the LMR [12], chemical off-equilibrium effects (determined by imposing pion number conservation) seem to be an important ingredient to understand dilepton production in the IMR.

\section{CONCLUSIONS}

In summary, we have evaluated dilepton spectra in central $\mathrm{Pb}(158 \mathrm{AGeV})+\mathrm{Pb}$ collisions as measured by the NA50 experiment. At intermediate masses the total spectra are composed of 3 major components: open charm decays, 
Drell-Yan annihilation and thermal radiation. Whereas the first one has been taken from NA50 event generator simulations, the Drell-Yan part has been calculated explicitly to both determine the data normalization and check the approximate acceptance of the NA50 detector. The thermal contribution is based on a realistic fireball expansion in accord with recent hadro-chemical analysis. Our main result is that the excess observed by the NA50 collaboration in the mass region $1.5 \mathrm{GeV}<M<2.5 \mathrm{GeV}$ can be explained by the thermal signal without invoking any anomalous enhancement in the charm production. The importance of the thermal yield quickly ceases beyond $M \simeq 2.5 \mathrm{GeV}$, thus not affecting the interpretation of $J / \Psi$ suppression effects - as opposed to scenarios invoking open charm enhancement. The contribution from the QGP phase turned out to be around $20-30 \%$.

Since the low-mass dilepton spectra from CERES/NA45 can be explained in the same framework (including medium effects in the low-lying vector mesons) a consistent picture of dilepton production at the full CERN-SpS energy seems to emerge. Similar conclusions have been reached in Ref. [20] where a more schematic dynamical evolution has been employed.

Let us finally address the question what can be done from the experimental side to further test our results. Firstly, a direct measurement of $D$ mesons will fix the open charm contribution to the dilepton spectra. Secondly, a dedicated (short) run of the NA50 experiment with reduced magnetic field to focus on the IMR would be most valuable. The resulting gain in acceptance for low- $q_{t}$ dileptons might quantitatively disentangle the thermal signal from hot/dense matter, putting model predictions under scrutiny and increasing the sensitivity to the very early stages of the collision.

\section{ACKNOWLEDGMENTS}

We are grateful for productive conversations with E. Scomparin, C. Gale, B. Kämpfer, C. Lourenço, H. Sorge and J. Wambach. One of us (RR) acknowledges support from the Alexander-von-Humboldt foundation as a Feodor-Lynen Fellow. This work is supported in part by the U.S. Department of Energy under Grant No. DE-FG02-88ER40388.

[1] E.V. Shuryak, Phys. Rep. 61 (1980) 71.

[2] E. Scomparin et al. (NA50 collaboration), Nucl. Phys. A610 (1996) 331c.

[3] P. Bordalo et al. (NA50 collaboration), Proc. of Quark Matter '99 (Torino, Italy, 10.-15.05.99), to be published in Nucl. Phys. A.

[4] N. Masera for the HELIOS-3 collaboration, Nucl. Phys. A590 (1995) 93c;

[5] G. Agakichiev et al., CERES collaboration, Phys. Rev. Lett. 75 (1995) 1272; Phys. Lett. B422 (1998) 405.

[6] S.D. Drell and T.-M. Yan, Phys. Rev. Lett. 25 (1970) 316; Erratum: ibid. p. 902.

[7] P. Lévai, B. Müller and X.-N. Wang, Phys. Rev. C51 (1995) 3326.

[8] Z. Lin and X.-N. Wang, Phys. Lett. B444 (1998) 245.

[9] C. Spieles et al., Eur. Phys. J. C5 (1998) 349.

[10] G.Q. Li and C. Gale, Phys. Rev. Lett. 81 (1998) 1572; Phys. Rev. C58 (1998) 2914.

[11] R. Rapp, G. Chanfray and J. Wambach, Nucl. Phys. A617 (1997) 472;

W. Cassing, E.L. Bratkovskaya, R. Rapp and J. Wambach, Phys. Rev. C57 (1998) 916.

[12] R. Rapp and J. Wambach, submitted to Phys. Lett. B, and hep-ph/9907502.

[13] R. Rapp and J. Wambach, Chiral Symmetry Restoration and Dileptons in Relativistic Heavy-Ion Collisions, to appear in Adv. Nucl. Phys. (1999), and hep-ph/9909229.

[14] M.C. Abreu et al. (NA50 collaboration), Phys. Lett. 410 (1997) 327.

[15] M. Glück, E. Reya and A. Vogt, Z. Phys. C67 (1995) 433.

[16] C.-Y. Wong, Phys. Rev. D30 (1984) 961.

[17] P. Braun-Munzinger and J. Stachel, Nucl. Phys. A638 (1998) 3c.

[18] E. Scomparin, private communication (1999).

[19] E. Scomparin et al. (NA50 collaboration), Proc. of the International Conference on 'Strangeness in Quark Matter '98' (Padova, Italy, 20.-24.07.98), J. Phys. G25 (1999) 235.

[20] K. Gallmeister, B. Kämpfer and O.P. Pavlenko, hep-ph/9908269. 